Revue d'histoire de l'Amérique française

6. REVUE D.HISTOIRE DE L'AMÉRIQUE FRANÇAISE

\title{
La construction domiciliaire à Québec, 1810-1820
}

\section{Jacques Bernier}

Volume 31, numéro 4, mars 1978

URI : https://id.erudit.org/iderudit/303651ar

DOI : https://doi.org/10.7202/303651ar

Aller au sommaire du numéro

Éditeur(s)

Institut d'histoire de l'Amérique française

ISSN

0035-2357 (imprimé)

1492-1383 (numérique)

Découvrir la revue

Citer cet article

Bernier, J. (1978). La construction domiciliaire à Québec, 1810-1820. Revue

d'histoire de l'Amérique française, 31(4), 547-561.

https://doi.org/10.7202/303651ar d'utilisation que vous pouvez consulter en ligne.

https://apropos.erudit.org/fr/usagers/politique-dutilisation/ 


\section{LA CONSTRUCTION DOMICILIAIRE À QUÉBEC 1810-1820 ${ }^{1}$}

JACQUES BERNIER

Montréal

Depuis quelques années, l'historiographie canadienne-française a élargi son champ d'investigation et les divers secteurs où s'exerce l'activité humaine retiennent de plus en plus l'attention des historiens québécois. En ce sens il apparaît normal que le travail, activité fondamentale de l'homme, revête une importance privilégiée. Ce thème a déjà donné lieu à quelques études importantes sur l'apprentissage, l'artisanat et la construction navale en Nouvelle-France ainsi que sur les mouvements ouvriers, la condition ouvrière et l'industrialisation au XIX ${ }^{\mathrm{e}}$ siècle $^{2}$. La période de la fin du XVIII ${ }^{\mathrm{e}}$ siècle et du début du $\mathrm{XIX}^{\mathrm{e}}$ siècle a été jusqu'ici assez peu explorée et en pratique seul l'apprentissage a suscité des études spécifiques ${ }^{3}$.

Pourtant, comme la ville de Québec est alors en pleine expansion (9000 habitants en 1805 et 15200 en 1819), tout laisse à penser que le travail ne manque pas et que le domaine de la construction y est florissant. C'est une époque où la ville s'étend pour loger une population locale qui s'accroît à un rythme élevé, recevoir les immi-

1 L'auteur tient à remercier J.-P. Wallot, P.-A. Linteau et N. Seguin pour leurs suggestions.

2 Pour une revue détaillée de la littérature sur l'histoire du travail au Québec voir: H. Espesset, J. P. Hardy et T. Ruddell, "Le monde du travail au Québec au $\mathrm{XVIII}^{\mathrm{e}}$ et au XIX ${ }^{\mathrm{e}}$ siècles: historiographie et état de la question», RHAF, 25, no 4 (mars 1972): 499-539 et $R$. Comeau «L'histoire ouvrière au Québec: quelques nouvelles avenues», RHAF, 28, n 4 (mars 1975): 579-583.

3 Voir à ce sujet: J.-P. Hardy et T. Ruddell, Les apprentis artisans à Québec 1660-1815 (Montréal, P.U.Q. 1977), 220 p., et P.-H. Audet, Apprenticeship in early nineteenth century Montréal, 1790-1812, thèse de maîtrise, (Université Concordia, 1975), 189 p. Depuis plusieurs années déjà, le Musée National de l'Homme s'intéresse aussi de près à l'histoire du travail au Québec: il rassemble des artefacts, subventionne des recherches et c'est dans le cadre de cette institution que s'inscrit notre étude. 
grants venus d'Europe et accueiflir les habitants et les villageois qui commencent à quitter la campagne pour la ville. Comme le secteur manufacturier n'est pas encore développé, Québec trouve dans la construction de maisons une source d'embauche appréciable.

$\mathrm{Au}$ moment de construire, le processus normal est d'aller devant notaire pour dresser des contrats appelés «marchés de construction». Ceux-ci constituent notre matériel principal et leur relevé a été grandement facilité par un travail préalable exécuté sous la direction de Christina Southam. Son équipe a répertorié d'une façon exhaustive tous les marchés de construction de la région de Québec conservés aux Archives Nationales du Québec à Québec pour la période entre 1800 et $1870^{4}$. Ces contrats sont nombreux, aussi nous avons dû procéder à un échantillonnage qui nous a amené à retenir trois années: 1810,1815 et 1820 , pour lesquelles nous avons examiné tous les contrats (soit 105 contrats). Le choix de ces 3 dates prises à 5 années d'intervalle permet d'éviter l'écueil de l'étude d'une période marquée par une conjoncture trop particulière et de mieux cerner une décennie dans sa globalité.

Ces archives concernent la construction civile et surtout résidentielle. Elles ne touchent pas la construction militaire, également importante, mais dont les papiers sont conservés aux Archives Publiques à Ottawa. Tous ces contrats de construction sont sensiblement rédigés suivant le même modèle. Après l'inscription de la date, le notaire procède toujours à la présentation des contractants. L'objet du contrat est alors précisé, suivi des conditions d'exécution du projet. On fixe ensuite la date de livraison et les modalités de paiement. Celles-ci varient souvent d'un contrat à l'autre mais les signataires s'entendent généralement pour que le dernier versement ne soit jamais effectué tant que le travail n'a pas été «déclaré parfait à dire d'experts». Le contrat est clos par des garanties qui assurent la protection des deux parties et une phrase vient ordinairement rappeler que "pour la nécessité de l'accomplissement du marché les parties obligent et hypothèquent tous leurs biens meubles et immeubles présents ou futurs».

Les données fournies par ces contrats commencent à éclairer certains aspects du monde de la construction domiciliaire et per-

4 C. Southam et al., Inventaire des marchés de construction passés à Québec entre 1800 et 1870 (Ottawa, Ministère des Affaires Indiennes et du Nord, 1974), 3 tomes. 
mettent de suivre le rythme d'accroissement des quartiers de la ville et de découvrir certains points de leur architecture. Grâce à elles nous pouvons aussi soulever le problème de la répartition des tâches selon les corps de métier et aborder celui de la division du travail selon les titres. Il est aussi possible de répondre à certaines questions d'ordre plus pratique comme: à combien s'élèvent les coûts des travaux? Comment se font les règlements entre parties? Quand construit-on et combien de temps durent les travaux? Enfin, on peut constater que s'il y a eu de grands changements dans les techniques de construction les termes des ententes ont évolué également, bien que d'une façon moins spectaculaire.

\section{1. Évolution des quartiers et quelques aspects architecturaux}

La ville de Québec présente, au début du $\mathrm{XIX}^{\mathrm{e}}$ siècle, un développement lent et nous ne comptons, pour les deux années inventoriées durant la première décennie (1800 et 1805$)$, que 5 contrats la première année et 7 la deuxième. Ce calme ne dure pas et dans la décennie suivante la ville connaît un essor soudain: on relève 39 contrats en 1810, 24 en 1815 et 42 en 1820 , soit un total de 105 contrats. Comme la population de la ville s'est accrue de 6000 habitants entre 1805 et 1819 , il est probable que ces chiffres n'incluent pas tous les travaux, ceci pour plusieurs raisons. Certaines personnes, fidèles à la coutume, préféraient certainement s'en tenir à des ententes orales moins coûteuses. D'autre part, les Anglo-Saxons, chez qui l'habitude de faire des contrats était beaucoup moins répandue que dans les pays influencés par le droit civil français, devaient continuer de pratiquer les «agreements » entre parties. Enfin l'on n'a pu retracer les papiers concernant la construction de maisons appartenant à des hauts fonctionnaires qui jouissaient de la possibilité de faire exécuter leurs travaux par l'armée 5 . Toutes ces restrictions $^{6}$ nous empêchent donc de considérer nos résultats comme absolument représentatifs du développement réel de la construction dans cette ville, mais ils en fournissent certainement une bonne

5 Information fournie par Christina Southam; on sait que les maisons Sewell et Goldworthy sur la rue Sainte-Anne furent ainsi construites. On sait aussi que de nombreux militaires, en plus de travailler comme maçons, menuisiers et charpentiers pour l'armée, louaient leurs services à des particuliers. Voir série C, Archives Publiques du Canada, Ottawa. travaux.

6 Plus, bien sûr, le fait que plusieurs durent effectuer eux-mêmes leurs 
approximation, surtout en milieu francophone. Ces contrats ne concernent pas tous de nouveaux bâtiments mais regroupent aussi des travaux de réparation et certains travaux marginaux. Au total, 67 d'entre eux impliquent des constructions nouvelles, 26 portent sur des travaux de réparation et de menuiserie, 10 sur des cheminées et des solages et les 9 autres, sur divers travaux.

Quand on regarde la répartition des contrats par quartier, on voit que l'activité se concentre principalement dans Saint-Roch (tableau 1). Ce quartier semble jouer le rôle de déversoir pour les migrants tant étrangers qu'autochtones ${ }^{7}$ et $53,7 \%$ des constructions nouvelles se font dans ce quartier. Quant au quartier Saint-Jean qui s'est surtout développé au XVIII ${ }^{\mathrm{e}}$ siècle son taux de croissance rejoint maintenant celui des vieux quartiers. Comme ces derniers, il semble avoir atteint un développement maximal et en 3 ans, il s'accroît seulement de 10 bâtisses contre 7 dans la Haute-Ville et 6 dans la Basse-Ville. En dehors de ces quartiers, l'activité est réduite et le quartier Saint-Louis tout comme la banlieue ne se développent pratiquement pas. À ces nouvelles constructions comprenant surtout des maisons, s'ajoutent quelques hangars, entrepôts et magasins. Ces édifices à caractère commercial, qui constituent la grande majorité des nouvelles bâtisses élevées en HauteVille, confirment de plus en plus la vocation commerciale de ce quartier.

Plus que tout autre caractéristique, c'est le matériau qui témoigne de la différence entre quartiers chic et quartiers populaires. Les quartiers riches, Haute-ville et Basse-ville, où demeurent les bourgeois, les membres des professions libérales et les administrateurs, restent fidèles à leur matériau premier, la pierre, tandis que la population ouvrière de Saint-Roch utilise un matériau moins onéreux, le bois. Mitoyen avec les vieux quartiers et avec Saint-Roch le quartier Saint-Jean, dans son nouveau développement, s'apparente davantage à ce dernier même s'il s'y fait encore quelques constructions en pierre.

Un autre trait distinctif entre ces quartiers tient dans la dimension de la surface habitable. La grandeur des constructions en bois est fort variable et si la moyenne se situe entre 500 et 1000 pieds

7 Sur la structure socio-spatiale de la ville de Québec, voir John Hare, «La population de la ville de Québec: 1795-1805», Histoire sociale, VII, (mai 1974): 23-42. 
TABLEAU 1

Répartition des travaux de construction effectués à Québec, 1810-1815-1820 (total)

\begin{tabular}{|c|c|c|c|c|c|c|c|c|c|c|c|}
\hline \multirow[t]{2}{*}{ Quartiers } & \multicolumn{6}{|c|}{$\begin{array}{c}\text { Travaux du bois } \\
74 \text { contrats }(80 \text { travaux })\end{array}$} & \multicolumn{5}{|c|}{$\begin{array}{l}\text { Travaux de maçonnerie } \\
31 \text { contrats ( } 32 \text { travaux) }\end{array}$} \\
\hline & 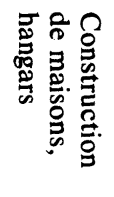 & 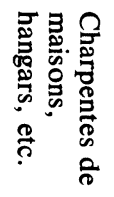 & 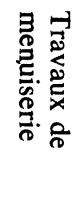 & 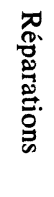 & 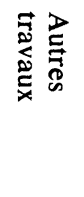 & $\stackrel{\overrightarrow{0}}{\ddot{D}}$ & 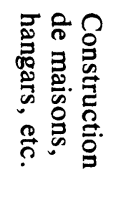 & 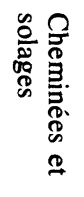 & 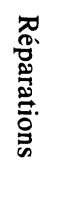 & 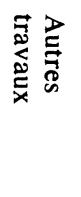 & $\stackrel{-1}{D}$ \\
\hline Haute-Ville & 1 & 4 & 1 & 2 & 2 & 10 & 2 & 1 & 2 & 1 & 6 \\
\hline Basse-Ville & 0 & 1 & 1 & 4 & 0 & 6 & $4^{3}$ & 2 & 3 & 0 & 9 \\
\hline Saint-Jean & 2 & 6 & 3 & 0 & 0 & 11 & 2 & 2 & 0 & 2 & 6 \\
\hline Saint-Roch & $12^{1}$ & $19^{2}$ & 4 & 1 & 2 & 38 & 0 & 5 & 2 & 0 & 7 \\
\hline Saint-Louis & 1 & 0 & 0 & 0 & 0 & 1 & 0 & 0 & 0 & 0 & 0 \\
\hline Banlieue & 0 & 2 & 1 & 0 & 1 & 4 & 1 & 0 & 1 & 0 & 2 \\
\hline Inconnus & 0 & 3 & 1 & 0 & 0 & 4 & 0 & 0 & 0 & 1 & 1 \\
\hline Total des contrats & 16 & 35 & 11 & 7 & $5^{4}$ & 74 & 9 & 10 & 8 & 4 & 31 \\
\hline Total des travaux & 18 & 39 & 11 & 7 & 5 & 80 & 10 & 10 & 8 & 4 & 32 \\
\hline
\end{tabular}

1 Un contrat comprend la construction de 3 maisons.

Deux contrats incluent la construction de 2 charpentes de maisons et un autre contrat de 3 charpentes de maisons.

3 Dont un contrat implique l'édification de 2 maisons.

4 Dont 2 pour faire une couverture en bardeaux ; un pour la construction d'un pont; un pour des réparations à la salle d'audience de la cour du banc du roi et un pour faire les échaffaudages pour la construction de la voûte de l'église Notre-Dame de Québec. 
carrés, cette dimension n'atteint parfois même pas les 300 pieds carrés. Saint-Roch, où la taille moyenne d'une maison atteint $\mathbf{7 8 3}$ pieds carrés, nous donne une bonne idée de la superficie des habitations dans un quartier construit en bois. Propriété de gens aisés, les maisons en pierre sont généralement plus grandes; toutes dépassent 1000 pieds carrés et certaines d'entre elles atteignent même les 2000 pieds carrés. Comparées à ces dernières les maisons en bois se révèlent donc petites puisque contrairement aux habitations en pierre, elles n'ont généralement qu'un seul étage. Matériaux bien différents dans leurs apparences et dans la façon dont on les travaille, la pierre et le bois donnent lieu à des constructions bien distinctes qui sont le reflet d'une différence non seulement architecturale mais aussi sociale entre les quartiers.

\section{L'organisation des corps de métiers}

Un autre type de question auquel nos sources nous permettent de répondre a trait à l'organisation du travaii et à la distributitioñ des tâches. Est-il possible de parler à cette époque de corps de métiers spécialisés, c'est-à-dire de saisir un cloisonnement des tâches entre les corps de métiers? D'autre part, dans un même métier, pouvons-nous déceler une division du travail en fonction des titres?

Les résultats obtenus à partir de ces contrats révèlent que les travaux de charpenterie sont presque tous (90\%) confiés à des spécialistes en charpenterie et que ceux de menuiserie reviennent dans une proportion presque aussi forte à des artisans de la menuiserie. Des interférences entre ces catégories apparaissent dans le secteur de la construction des maisons où, sur 15 contrats, 5 sont exécutés par des charpentiers, 4 par des charpentiers-menuisiers, 3 par des menuisiers, 1 par un menuisier-charpentier et 2 par des habitants. Bien que les charpentiers soient en plus grande demande que les menuisiers cela n'inclut toutefois pas une grande différence dans les travaux à exécuter. Les maisons construites par les uns et les autres ont sensiblement la même superficie et sont à un seul étage. On remarque tout de même que les travaux plus importants sont confiés exclusivement aux charpentiers qui se voient attribuer la construction de toutes les demeures de plus de 900 pieds carrés. En somme le monde des ouvriers du bois est un monde ordonné où les charpentiers travaillent aux charpentes, les menuisiers à la menuiserie et les deux à la construction des maisons, même si les charpentiers restent, dans nos actes, les plus en demande dans ce secteur. 
À l'intérieur du corps des menuisiers et des charpentiers existet-il une différence entre le travail des maîtres, des «entrepreneurs ", et des simples artisans? En charpenterie, ces derniers exécutent à eux seuls près de la moitié des contrats. Ils dépassent de loin les autres groupes qui en obtiennent respectivement: les maîtres charpentiers, $18 \%$, les charpentiers-menuisiers, $18 \%$, et les «entrepreneurs en charpenterie», moins de 7\%. Quant aux tâches, aucune d'elles ne peut être considérée comme le domaine exclusif d'une catégorie de travailleurs; tous exécutent divers travaux bien que les «entrepreneurs en charpenterie» signent souvent les contrats les plus importants. Dans le secteur de la menuiserie, les simples menuisiers sont aussi en demande que les maîtres et les uns comme les autres s'appliquent aux mêmes tâches. Ainsi, si une division du travail entre charpentiers et menuisiers ressort assez clairement, la distinction que laissait pressentir le titre de maître ne se vérifie pas dans l'attribution des tâches et l'on s'explique mal ce que sous-tend ce titre.

Contrairement au secteur du bois où l'on a vu l'intervention de quelques habitants, les travaux de maçonnerie, domaine plus spécialisé, sont toujours confiés à des maçons. Sur les 31 contrats relevés pour ces 3 années, les maîtres maçons en concluent 16 , les maçons 7 et les «entrepreneurs en maçonnerie» 7. Les maîtres maçons n'exécutent pas seulement le plus grand nombre de tâches mais aussi les plus diverses: maisons, hangars, rénovations, solages, cheminées, fours. De leur côté les «entrepreneurs en maçonnerie» sont surtout spécialisés dans les travaux de dimensions importantes comme les hangars et les maisons de grande taille. Les simples maçons quant à eux font des travaux plus modestes et plus faciles comme certains travaux de rénovation, des solages et des cheminées ainsi que certains travaux d'excavation. En opposition aux artisans du bois qui travaillent le plus souvent seuls, les maçons ont davantage tendance à collaborer et dans plus de $43 \%$ des contrats les ouvriers sont associés.

Pratiquement absents, dans nos contrats, du secteur du bois (5/74) les anglophones sont mieux représentés dans celui de la maçonnerie où ils signent 5 des 31 contrats $^{8}$. Ils y jouent de plus

8 Les anglophones qui ne forment pas $10 \%$ de la main-d'œuvre de la maçonnerie à Québec en 1805 en viendront en 1831 à constituer près du tiers des ouvriers de ce secteur (contre $16 \%$ des menuisiers et $19 \%$ des charpentiers). Voir F. Ouellet, «Structure des occupations et ethnicité de Québec et de Montréal, 1819-1844», Éléments d'histoire sociale du Bas-Canada (Montréal, H.M.H., 1972), 177-202. 
un rôle important et les 3 plus gros contrats de maçonnerie et de plâtre leur reviennent. L'un se rapporte à la construction d'une auberge de 3 étages, l'autre à l'édification d'une maison de 2988 pieds carrés sur la rue Saint-Paul et enfin, le plus important se rapporte à l'édification de la voûte de la nef de l'église Notre-Dame de Québec.

L'organisation du monde des ouvriers de la maçonnerie diffère donc de celle des ouvriers du bois car le travail y est spécialisé, la distribution des tâches plus hiérarchisée, et le travail en coopération, beaucoup plus fréquent.

\section{Les clauses des contrats}

Parmi les premières composantes de cette relation entre client et entrepreneur s'inscrivent deux éléments essentiels consécutifs à la nature même des travaux de construction et qui portent sur les matériaux et les hommes. Aussi étrange que cela puisse apparaître maintenant, les deux parties s'obligent, suivant les cas, à fournir des ouvriers, des matériaux et même à exécuter certains travaux.

Pour les travaux du bois, les apports de part et d'autre consistent surtout dans du matériel divers (bois, clous, madriers, lambourdes, vitres, etc.). À cette participation en matériaux s'en ajoute souvent une en hommes. Celle-ci peut être continue durant toute la durée des travaux ou n'intervenir qu'à l'occasion d'une étape particulière dans l'exécution du travail comme, par exemple, lors de la levée des charpentes. Il n'est pas rare non plus que l'employeur fournisse de la nourriture en plus du salaire et que, de son côté, le demandeur apporte le bois sur les lieux de travail. Dans près de $36 \%$ des cas, exécutants et demandeurs ont des obligations respectives tandis que dans $27 \%$ des cas ce sont seulement les demandeurs, et dans $33 \%$ uniquement les entrepreneurs qui en assument la responsabilité.

En maçonnerie, la répartition est plus inégale. Dans $13 \%$ des contrats seulement, les demandeurs font des apports et dans $36 \%$, ce sont les entrepreneurs. Les apports mutuels sont ici plus élevés que dans les travaux du bois et atteignent près de la moitié du total des contrats. Là aussi il s'agit de contributions en matériaux (pierre, mortier, brique, chaux, sable) et en hommes, mais ici ces derniers sont presque toujours fournis et nourris par les employeurs. Cet écart entre le nombre de demandeurs-pourvoyeurs dans le bois comparativement au nombre plus faible obtenu dans la maçonnerie 
peut s'expliquer par une différence d'accès aux matériaux. En effet, alors que les carrières de pierre de Beauport sont éloignées et d'exploitation coûteuse, le bois entoure la ville et peut être facilement obtenu de l'habitant par le client. Le commerce du bois est alors aussi très florissant dans le Bas-Canada et Québec, port par lequel transite une grande partie du bois en direction de l'Angleterre, offre des facilités d'achat de ce matériau. D'autre part, comme le travail de la pierre nécessite une plus grande spécialisation que celui du bois, on peut penser que c'est pour cette raison que les maçons préfèrent souvent choisir eux-mêmes leurs aides.

Les modes de rétribution varient beaucoup d'un contrat à l'autre et le montant versé à l'entrepreneur est fonction des apports comme de l'importance et de la qualité du travail à exécuter. Les situations où les deux parties font des apports sont les plus délicates à démêler, car il est difficile d'évaluer la contribution de chacune. Par contre, les contrats où une seule des parties est la pourvoyeuse permettent de faire une évaluation plus précise, et nous ne retiendrons que ces cas. Quant le demandeur est aussi le fournisseur, il semble qu'on donne rarement plus de 20 livres et rarement moins de 10 livres pour la construction d'une charpente de maison moyenne à un étage. Pour des charpentes de maison plus importantes, ces sommes peuvent doubler, voire quadrupler, et pour des charpentes de hangar ayant entre 1500 et 2500 pieds carrés, elles se situent généralement entre 30 et 40 livres. Lorsque l'artisan est aussi le fournisseur des matériaux, ces sommes s'élèvent à peu près du double et se situent pour les charpentes de maisons ordinaires entre 20 et 40 livres et pour les hangars autour de 75 livres. S'il s'agit de la construction d'une maison complète, cette rémunération varie entre 15 et 60 livres quand les matériaux sont fournis par le client et elle dépasse le plus souvent les 100 livres quand cette responsabilité incombe à l'artisan. Pour les travaux de menuiserie et de rénovation, l'échelle est beaucoup moins précise. Elle varie en fonction de l'importance et de la difficulté des travaux et on peut en trouver aussi bien à quelques livres qu'à 30,60 ou même 100 et plus. Une caractéristique propre aux ouvriers de la maçonnerie provient de ce que leur salaire est ordinairement évalué à la toise. Ceci se vérifie surtout pour la construction des maisons et des hangars qui tous suivent ce mode de paiement. On le retrouve également dans presque la moitié des marchés de solage et de cheminée mais il n'intervient que rarement dans les rénovations. Le prix de la toise varie ordinairement de 2 à 3 livres quand l'entrepreneur assure lui- 
même la fourniture des matériaux et dans ce cas, il peut recevoir entre 200 et 1000 livres, selon les dimensions et le nombre d'étages, pour la construction d'une maison en pierre. Quand les matériaux ne sont pas fournis par l'entrepreneur, le prix de la toise se situe alors aux environs d'une livre. Dans les cas où une somme précise est fixée à l'avance, l'ouvrier qui prend à charge les matériaux reçoit habituellement pour fabriquer un solage et 2 cheminées (travaux qui vont souvent de pair) entre 50 et 70 livres, et jamais moins de 10 livres pour une réparation. La lecture de ces chiffres fait ressortir qu'un entrepreneur qui construit une maison en pierre dont il apporte tous les matériaux exige approximativement de 6 à 8 fois plus cher qu'un entrepreneur qui dans les mêmes conditions érige une maison en bois. Quant aux travaux difficiles comme les solages et les cheminées en pierre, ils peuvent souvent atteindre le prix d'une petite maison en bois. La pierre apparaît donc comme un matériau coûteux, très peu accessible et qui n'est utilisé comme nous l'avons déjà signalé que dans les quartiers riches.

La modalité de paicment la plus répandue, tant chez les ouvriers du bois que chez ceux de la maçonnerie, consiste en des versements échelonnés à partir de dates précises fixées à l'avance ou en raison de l'évolution des travaux. Ainsi, $73 \%$ des contrats de maçonnerie et près de $40 \%$ des contrats du bois stipulent ce mode de paiement. Dans quelques cas, ces versements échelonnés sont précédés d'un paiement initial au moment de la signature du contrat devant le notaire. Il existe aussi d'autres genres de paiement mais ils sont en pratique peu répandus. Ce sont ceux où l'argent est versé à la fin ou après la réalisation du contrat; ceux, très rares, où le forfait est versé avant le début des travaux, et ceux enfin, également fort peu nombreux où le paiement est fait en nature. Une grande distinction toutefois est à souligner entre les ouvriers de la pierre et les ouvriers du bois: ces derniers disposent (dans plus de $32 \%$ des contrats) d'un mode de paiement qui n'est aucunement en vigueur chez leurs confrères maçons ${ }^{9}$ et qui consiste en un premier versement, généralement inférieur mais jamais supérieur à la moitié du total, suivi d'un dernier à la fin des travaux. Ce type de paiement qui n'existe que chez les ouvriers du bois et qui n'est le fait ni de gros contrats ni de travaux particuliers, doit être mis en relation avec les modalités d'achat du matériel. Ces achats, dans

9 Lesquels, comme nous l'avons vu, sont presque toujours payés au fur et à mesure des travaux. 
le secteur du bois, se font probablement d'une manière plus globale avant les travaux alors que pour la pierre ils ont lieu d'une façon plus échelonnée durant toute la durée de l'ouvrage. La somme d'argent versée au départ sert alors à couvrir une partie des frais engagés dans l'achat du bois.

La date de la remise du travail permet de dégager quelques éléments intéressants. Grâce à elle, nous pouvons connaître et le temps d'exécution et la période d'accomplissement de chaque type de travail. La durée d'un grand nombre de contrats pour des travaux en bois ou en maçonnerie ne dépasse pas 4 mois et $50 \%$ d'entre eux sont exécutés dans cette limite de temps. Ce pourcentage peut, du reste, être facilement relevé car bon nombre de contrats dont la durée excède 6 mois, furent signés au début de l'hiver et achevés à la fin du printemps (surtout au mois de mai); et il est probable que si ces travaux n'avaient pas été effectués durant la dure saison, ils auraient pu être livrés dans un temps plus court. Peu de travaux dépassent ce laps de temps excepté quelques tâches de grande envergure et ceci en particulier dans les ouvrages de maçonnerie. Dans le secteur du bois, la construction des maisons requiert le plus de temps et aucune ne peut être élevée en moins de 2 mois, tandis que la construction des charpentes varie entre 1 et 2 mois. Dans le secteur de la pierre, excepté les maisons qui demandent souvent 4 mois pour être bâties, les autres travaux ne durent eux aussi généralement pas plus de 2 mois.

Comme on peut s'en douter, la meilleure période pour faire ces travaux se situe surtout durant la belle saison, c'est-à-dire de mai à novembre. Ceci est particulièrement vrai pour les travaux de maçonnerie où tous les contrats de maison, de cheminées, de solages et (bien que moins régulièrement) de rénovations sont signés et exécutés durant les mois sans gel. Cette constatation s'applique cependant moins au bois, lequel ne craint pas le froid, et si la plupart des contrats de charpentes sont des contrats d'été, ceux des maisons sont indifféremment signés à n'importe quel moment de l'année mais arrivent ordinairement à terme à la belle saison. À une certaine limite de temps s'ajoutent aussi des exigences de qualité dans le travail qui, suivant le désir du client, pourront être appréciées et confirmées par des «experts et gens à ce connaissant».

Les clauses des contrats, comme nous avons pu le constater, diffèrent dans le secteur du bois et de la maçonnerie. Dans ce dernier secteur, les clients apportent moins souvent leurs matériaux car 
les maçons préfèrent les choisir eux-mêmes en raison de leurs habilités personnelles et en fonction de leurs connaissances des pierres qui, suivant les catégories, se prêtent mieux à certains travaux qu'à d'autres. Ces aspects sont moins importants dans le domaine du bois et c'est probablement ce qui explique que davantage d'artisans aient pu laisser le soin de l'achat du bois aux clients. Quant aux rémunérations, en raison de la compétence qu'exige le travail de la pierre, il apparaît normal que, pour un temps égal, les travaux de maçonnerie soient relativement plus payants que ceux de la menuiserie et de la charpenterie. Enfin, on peut vraisemblablement rendre compte des modalités différentes de paiement par le fait que les ouvriers du bois achètent leurs matériaux au début des travaux alors que les maçons en font l'acquisition tout au long de l'ouvrage.

\section{Signatures et lieux d'habitation}

Il aurait été intéressant, au terme de cette étude, d'apporter quelques éclaircissements sur la vie de travail et la vie quotidienne de ces artisans. Étaient-ils regroupés en corporation? Quels étaient les rapports entre artisans et journaliers? Quelles étaient les heures quotidiennes de travail? Quelle était la situation de l'emploi? Étaientils contraints à une grande mobilité ? Avaient-ils des protections en cas d'accidents? Quel était leur niveau de vie par rapport à celui des autres artisans et des autres groupes sociaux ? Voilà autant de questions intéressantes qui resteront ici à peu près sans réponse, car le contenu de nos documents est pratiquement muet sur ces sujets. Il est cependant deux points sur lesquels ces contrats nous permettent de dire quelques mots: leur lieu d'habitation et leur capacité à signer.

L'adresse qui donne le nom du village ou le quartier d'habitation est habituellement citée dans les marchés sauf dans $20 \%$ des cas où l'on indique seulement: «originaire de la ville Québec». La comparaison entre les artisans habitant la ville ${ }^{10}$ et ceux vivant hors de la ville, révèle que plus de $86 \%$ habitent à l'intérieur des murs contre près de $13 \%$ à l'extérieur. Les charpentiers forment le gros de cette population extra-muros $(71 \%)$ qui vient des villages avoisinants comme Beauport, Lévis, l'Ancienne-Lorette, Saint-Nicolas et de paroisses plus éloignées comme Louiseville et Trois-Rivières. À l'intérieur de la ville, la distribution des artisans par quartier met en

10 Haute-ville, Basse-Ville, Saint-Jean et Saint-Roch. 
évidence l'importance de Saint-Roch qui groupe à lui seul plus de $37 \%$ des artisans. Dans nos contrats les charpentiers s'y retrouvent à près de $47 \%$, les maçons à plus de $36 \%$ et les menuisiers à $25 \%{ }^{11}$. Après Saint-Roch le quartier Saint-Jean est ensuite celui qui en abrite le plus, tandis que la Haute-Ville et la Basse-Ville en reçoivent très peu.

Cette différence sociale que l'on remarque dans le lieu d'habitation se retrouve aussi dans le degré d'alphabétisation et même si la signature n'est pas toujours un indice certain d'alphabétisation, elle témoigne au moins de la volonté de faire face à ses obligations et à un souci d'identification sur le plan social. Sur seulement $42 \%$ des 105 contrats les artisans apposèrent leur signature. Les charpentiers arrivent au plus bas rang avec un pourcentage de signataires de $32 \%$ devancés par les maçons qui obtiennent $36 \%$. Les menuisiers se détachent nettement de ces 2 groupes «d'analphabètes» et $60 \%$ d'entre eux furent capables de signer. Précisons encore qu'à l'intérieur de chacun de ces métiers un autre regroupement peut s'établir entre "entrepreneurs ", maîtres et simples artisans. Les «entrepreneurs» font montre de la plus grande aptitude et on trouve chez eux des signataires dans 7 cas sur 12. Les maitres viennent ensuite, suivis en dernier des simples artisans. Ces conclusions auxquelles nous mènent ces observations sur le degré d'alphabétisation rejoignent d'ailleurs des résultats obtenus lors d'une recherche sur les intérieurs domestiques des menuisiers et des charpentiers de la région de Québec entre 1810 et $1819^{12}$. Il en ressortait que les menuisiers étaient plus fortunés que les charpentiers et qu'ils possédaient des maisons plus grandes et mieux équipées. Leurs prisées d'ailleurs étaient non seulement plus élevées que celle des charpentiers mais les menuisiers étaient également propriétaires de plus de possessions et de terrains et les dettes de leurs créanciers étaient beaucoup plus considérables que celles des créanciers des charpentiers.

11 Rappelons toutefois que ces pourcentages ne doivent pas être pris à la lettre vu le nombre élevé d'artisans dont l'origine précise n'est pas connue. Ils confirment cependant en gros le phénomène observé par J. Hare entre 1795 et 1805 , à savoir le mouvement migratoire de la population ouvrière vers les quartiers SaintRoch et Saint-Jean. Voir J. Hare, op. cit., 34.

$12 \mathrm{~J}$. Bernier, Les intérieurs domestiques des menuisiers et charpentiers de la région de Québec, 1810-1819 (Ottawa, Musées Nationaux du Canada, 1978). 


\section{Conclusion}

En conclusion, certaines données méritent d'être rappelées. Le quartier Saint-Roch est, entre 1810 et 1820, en pleine expansion et c'est là que se déroule l'essentiel de l'activité de la construction. Les maisons élevées dans ce quartier obéissent à certaines caractéristiques propres: elles sont toutes en bois, de dimensions modestes et n'ont ordinairement qu'un seul étage. Les quelques maisons en pierre citées dans les contrats sont érigées dans les vieux quartiers qui abritent une population plus fortunée. Ces habitations sont faites en un matériau plus difficile à travailler, sont aussi beaucoup plus grandes, plus longues à construire, et pour ces raisons, coûtent plus cher. Comme ils sont anciens, ces quartiers centralisent aussi l'essentiel des travaux de rénovation.

Des différences importantes ressortent également à l'intérieur des corps de métier. Dans les métiers du bois, charpentiers et menuisiers font en règle générale des travaux qui leurs sont propres. Tous deux cependant travaillent à élever des maisons, mais les charpentiers ont la charge des constructions les plus importantes. On ne remarque pas de tâches particulières réservées aux maîtres ou aux simples artisans, sauf pour les «entrepreneurs en charpenterie» qui se voient ordinairement confier les tâches les plus grosses. Au point de vue social, les menuisiers se distinguent des charpentiers par un plus grand taux d'alphabétisation, des habitations meilleures situées dans des quartiers moins modestes et une moins grande mobilité géographique.

Maçons et ouvriers du bois sont rémunérés suivant les mêmes principes mais ces derniers reçoivent des sommes initiales et terminales plus importantes. Chez les maçons une hiérarchie apparaît plus clairement, suivant la difficulté des travaux, entre «entrepreneurs », maîtres et non-maîtres et le travail en collaboration y est répandu. Les quartiers où ils habitent tout comme leur niveau d'alphabétisation les situent socialement dans une position intermédiaire entre les menuisiers et les charpentiers. Telles sont donc brièvement résumées quelques caractéristiques principales qui peuvent se dégager de cette recherche.

Un des buts poursuivis par cet article aura été d'explorer une source peu utilisée par les historiens. Cette source évidemment, comme toute autre source unique, est fragmentaire et ne rend certainement pas compte de toute l'activité de la construction à Québec durant cette période. Son utilisation aura toutefois servi à mettre 
l'accent sur le développement géographique et architectural de la ville et à mieux connaître certains aspects du monde du travail.

Rappelons enfin que l'activité de la construction a suscité peu de monographies dans l'historiographie québécoise et qu'elle serait pourtant digne d'un meilleur sort pour plusieurs raisons: d'abord parce qu'elle constitue un bon indice de l'activité économique d'un pays (ne dit-on pas en effet que lorsque la construction va bien, le pays se porte bien) et parce qu'elle centralise dans toute société en développement une partie importante du capital et de la maind'œuvre. D'autre part on peut aussi, sur un autre plan, invoquer le caractère nécessaire de cette activité qui touche aux besoins les plus immédiats de l'homme. Se loger ne constitue-t-il pas en effet une des premières nécessités de l'homme après l'alimentation. De nombreuses études utiles pourraient pourtant être menées à son sujet. Il serait intéressant par exemple de situer l'importance de la construction par rapport aux autres secteurs de l'activité économique. On pourrait aussi essayer de dégager les faits marquants de cette activité en mettant d'abord en relief son caractère traditionnel puis les transformations qu'elle connaîtra à partir (probablement) de la deuxième moitié du $\mathrm{XIX}^{\mathrm{e}}$ siècle. On pourrait encore faire ressortir l'évolution de l'organisation générale de cette activité, faire voir les réseaux de transmission du savoir (transmission orale, apprentissage) et sur un plan plus concret toucher de plus près aux aspects techniques: matériaux, outils, inventions. Enfin, ultérieurement, une question fascinante à aborder serait celle des industries annexes à celle du bâtiment et qui évoluent en étroite relation avec elle: les fabriques de chaux, de plâtre et de ciment, les briqueteries, les verreries, les carrières, les scieries, etc... On verrait probablement, en y incluant ces activités subsidiaires et la construction militaire, que ce secteur constituait l'une des plus importantes activités dans la ville de Québec d'alors. 